\title{
Multiple-Beams Splitter Based on Graphene
}

\author{
Xiao Bing Li, Hong Ju Xu, Wei Bing Lu, and Jian Wang
}

State Key Laboratory of Millimeter Waves, School of Information Science and Engineering, Southeast University, Nanjing 210096, China

Correspondence should be addressed to Wei Bing Lu; wblu@seu.edu.cn

Received 28 March 2016; Accepted 5 June 2016

Academic Editor: Zhihao Jiang

Copyright (C) 2016 Xiao Bing Li et al. This is an open access article distributed under the Creative Commons Attribution License, which permits unrestricted use, distribution, and reproduction in any medium, provided the original work is properly cited.

Due to its tunability of conductivity, graphene can be considered as a novel epsilon-near-zero (ENZ) material. Based on this property, we propose a wave splitter using graphene. Simulation results show that the circular surface plasmon polariton waves excited by a point source can be transferred to narrow beams through a graphene-based wave splitter, which is formed by a polygonal contour of the ENZ graphene layer. The number of beams can be easily controlled by adjusting the shape of the polygonal ENZ graphene layer, and the operation frequency can also be chosen.

\section{Introduction}

In recent years, there has been a great deal of interest in physics and engineering of artificially constructed metamaterials, due to their exciting properties caused by unconventional values of permittivity $(\varepsilon)$ or permeability $(\mu)[1-6]$. In particular, much attention has been focused on structures for which the real part of one or both of the constitutive parameters approaches zero [2]. These structures have been used to form interesting devices such as highly directive antennas [7] and compact resonators [8]. It is proved that materials with epsilon-near-zero (ENZ) may be directly found in nature in infrared and optical frequency band [3]. A well-known example is electron gas in which the current created by the drift of free electrons effectively interacts with the radiation as continuous medium characterized by a Drude-type dispersion model that has near-zero $\varepsilon$ around its plasma frequency [1]. In infrared and optical frequencies, some low loss noble metals like silver and gold, semiconductors (e.g., indium antimonide) [9], and polar dielectrics like silicon carbide $(\mathrm{SiC})[10]$ may behave as ENZ materials near their plasma frequencies. However, due to their dispersive properties, such ENZ materials only work well near their plasma frequencies. So, finding new ENZ materials at desired frequencies is important to design functional devices.

Graphene [11-17], due to its various intriguing properties, has been considered as one of the promising optical materials in the future $[16,18]$. The one-atom-thick graphene is characterized by a surface conductivity $\left(\sigma_{g}=\sigma_{g, r}+i \sigma_{g, i}\right)$, which can be computed by the Kubo formula [10, 19]:

$$
\begin{gathered}
\sigma\left(\omega, \mu_{c}, \Gamma, T\right)=\frac{j e^{2}(\omega-j 2 \Gamma)}{\pi \hbar^{2}}\left[\frac{1}{(\omega-j 2 \Gamma)^{2}}\right. \\
\cdot \int_{0}^{\infty} \varepsilon\left(\frac{\partial f_{d}(\varepsilon)}{\partial \varepsilon}-\frac{\partial f_{d}(-\varepsilon)}{\partial \varepsilon}\right) d \varepsilon \\
\left.-\int_{0}^{\infty} \frac{f_{d}(-\varepsilon)-f_{d}(\varepsilon)}{(\omega-j 2 \Gamma)^{2}-4(\varepsilon / \hbar)^{2}} d \varepsilon\right],
\end{gathered}
$$

where $\omega$ is the radian frequency, $\Gamma$ is the phenomenological scattering rate, $T$ is the temperature, $\mu_{c}$ is the chemical potential, $e$ is the electron charge, $\hbar=h / 2 \pi$ is the reduced Planck constant, $f_{d}(\varepsilon)=\left(e^{\left(\varepsilon-\mu_{c}\right) / k_{B} T}+1\right)^{-1}$ is the Fermi-Dirac distribution, and $k_{B}$ is Boltzmann's constant. Importantly, the conductivity of graphene can be changed via tuning the chemical potential, which is controlled by gate voltage, electric field, magnetic field, and/or chemical doping [20, 21]. According to the relationship between equivalent complex permittivity and conductivity of the $\Delta$-thick graphene layer (graphene has a very small thickness $\Delta$ ), given in [22], we can tune the equivalent complex permittivity of the oneatom-thick graphene layer. In addition, graphene can support 
the highly confined transverse-magnetic (TM) surface plasmon polariton (SPP) wave if and only if $\sigma_{g, i}>0$ (in other words, $\left.\operatorname{Re}\left(\varepsilon_{g, \text { eq }}\right)<0\right)[16,23]$. Based on the above conclusions, graphene shows the potential to construct transformational metamaterials on a one-atom-thick surface.

In graphene, the chemical potential depends on the carrier density $(n)$ which can be modified dynamically by applying a gate voltage [24]. The relation between $\mu_{c}$ and $n$ can be described as the following formula: $\left|\mu_{c}\right|=\hbar v_{F}(\pi n)^{1 / 2}$, where $v_{F} \approx 1 \times 10^{6} \mathrm{~m} \mathrm{~s}^{-1}$ is the Fermi velocity, $n$ is given by $n=\left(n_{0}^{2}+\alpha^{2}|\Delta V|^{2}\right)^{1 / 2}, n_{0}$ is the residual carrier concentration, $\alpha$ is the gate capacitance effected by the specific electrode configuration, $|\Delta V|=\left|V_{\mathrm{CNP}}-V_{g}\right|$, with $\left|V_{\mathrm{CNP}}\right|$ being the charge neutral gate voltage, and $V_{g}$ is the applied gate voltage. The chemical potential of graphene can be controlled using an ion-gel top gate [25], which allows a large doping range through low electrostatic voltage. Through varying the chemical potential, we can find an equivalent complex graphene permittivity value at the working frequency, the real part of which is close to zero, either positive or negative. Here we choose negative ENZ values in order to support the propagation of SPP waves on the graphene. In this paper, we propose a tunable wave coupler based on a single sheet of graphene. The low wave number of propagation characteristics of such materials implies that the phase variation of the electromagnetic fields is negligible over a physically long distance, providing the possibility of manipulating the phase fronts into a desired pattern by controlling the shape of the interfaces of the ENZ material.

In the infrared frequency band, the near-zero epsilon values can be obtained via changing the gate voltage, which is related to the chemical potential [26]. Figure 1 shows the relation between the chemical potential and the real part of graphene's equivalent dielectric permittivity at different frequencies with $T=3 \mathrm{~K}$ and $\Gamma=0.43 \mathrm{meV}$. As can be seen, when the working frequency increases, the chemical potential corresponding to zero permittivity rises, implying that the wave coupler we have designed can also operate at other frequencies through tuning the gate voltage.

\section{Simulation Model}

Figure 2 shows the simulation model of the wave splitter based on graphene. The radius of the inner cylinder is denoted as $R 1$, the apothem of the polygon is denoted as $R 2$, and the diameter of proposed wave splitter is $D$. The red zone corresponds to the ENZ graphene layer. In this area, the real part of graphene's dielectric permittivity approaches zero. In other areas, it can be defined as a negative value to support the propagation of SPP waves. According to [17], uneven ground plane can be used to create inhomogeneous conductivity or permittivity pattern along graphene layer. This is schematically shown in Figure 3(a). Highly doped silicon substrate with uneven height profile serves as the ground plane. The distance between the ground plane and graphene can be filled up with a regular dielectric spacer, for example, silicon oxide. In the numerical simulation, a point source on top of the graphene layer is utilized for

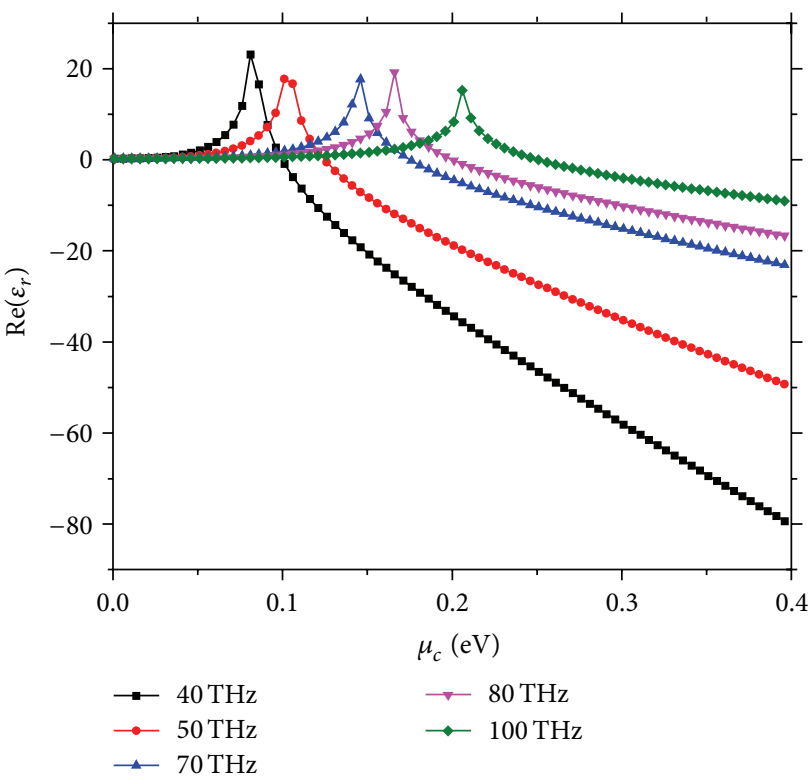

FIGURE 1: Relation between chemical potential and real part of graphene's equivalent dielectric permittivity at different frequencies with $T=3 \mathrm{~K}$ and $\Gamma=0.43 \mathrm{meV}$.

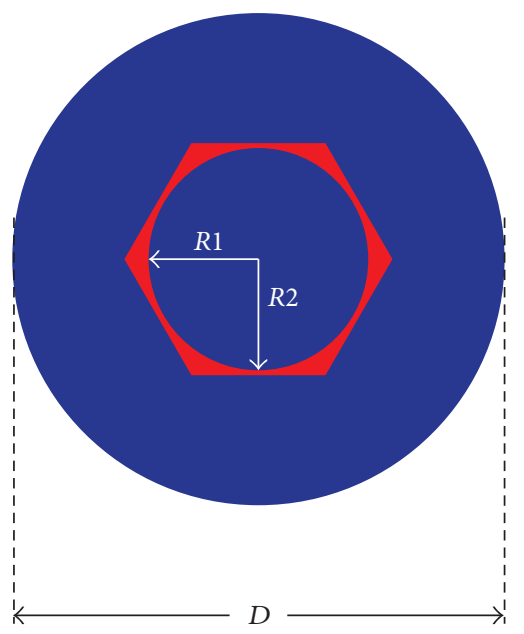

FIGURE 2: Simulation model of the wave splitter based on graphene. The red zone is the ENZ graphene layer, and the blue zone is the graphene with negative dielectric permittivity to support the propagation of SPP waves.

exciting the circular SPP waves. The distance between the source and the wave splitter is $H=10 \mathrm{~nm}$, as shown in Figure 3(b). To satisfy the wave vector matching condition, the evanescent fields emitted from the infinitesimal dipole are used to excite the circular SPP waves on the graphene. So, the distance between the dipole and the graphene is much smaller than the working wavelength. In our design, the distance is chosen to be less than a thousand of the working wavelength. 


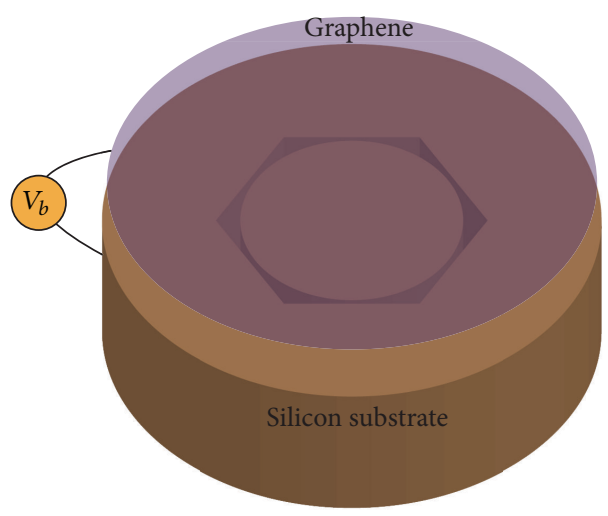

(a)

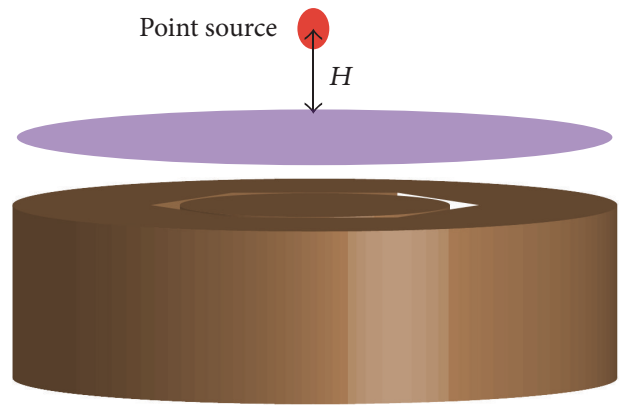

(b)

FIGURE 3: (a) Sketch of our proposed one-atom-thick wave splitter, which consists of a single sheet of graphene with inhomogeneous conductivity or permittivity distributions. (b) A point source on top of the graphene layer is used to excite circular SPP waves. The distance between the source and the wave splitter is $H=10 \mathrm{~nm}$.

\section{Numerical Results and Discussions}

In this paper, we use a full-wave electromagnetic simulator software, CST Microwave Studio [27], to obtain threedimensional numerical results. The numerical calculations use frequency-domain solver, performed with adaptive tetrahedral meshing and open boundary conditions for a freestanding graphene in vacuum without ground plane. An infinitesimal dipole is used as a point source to generate the circular SPP waves. To confirm the effectiveness of the proposed wave splitter based on graphene, we take three-, four-, five-, and six-beam splitter as specific examples. In all simulations, the working frequency is chosen as $50 \mathrm{THz}$ and we set $R 1=90 \mathrm{~nm}, R 2=95 \mathrm{~nm}$, and $D=400 \mathrm{~nm}$. The thickness of graphene is taken as $1 \mathrm{~nm}$. According to Figure 1, we can obtain the equivalent complex permittivity $\varepsilon_{r, \text { eq }}=-2.28 \times 10^{-4}+i 0.2178$ with $T=3 \mathrm{~K}, \Gamma=0.43 \mathrm{meV}$, and $\mu_{c}=0.12155 \mathrm{eV}$, which acts as ENZ graphene layer. However, the loss of the ENZ graphene area is large. As a consequence, when the graphene layer is used as ENZ material, the geometrical size of ENZ region should be set very small in order to reduce the propagation loss.

The chemical potential of the other background graphene layer is chosen as $\mu_{c}=0.1799 \mathrm{eV}$, corresponding to the complex permittivity $\varepsilon_{r, \text { eq }}=-15.05+i 0.1511$. It should be emphasized that, for the background graphene layer, any chemical potential larger than $0.129 \mathrm{eV}$ (when $\mu_{c}>0.129 \mathrm{eV}$, $\operatorname{Re}\left(\varepsilon_{r}\right)<0$ and $\left.\left|\operatorname{Re}\left(\varepsilon_{r}\right)\right|>1\right)$ is capable of supporting the SPP waves propagation. Figure 4(a) shows the snapshot of the $z$-component electric field $E_{z}$ distribution of the TM SPP wave of the graphene-based wave splitter, of which the outer contour of the ENZ graphene is triangle. When the circular SPP waves propagate through the ENZ graphene region, three beams of the almost linear SPP waves emerge. When the outer contour of the ENZ graphene layer is a square with length of $R 2$, a four-beam wave splitter is realized, as shown in Figure 4(b). In the same way, five- and six-beam wave splitters have been designed and demonstrated. The simulation results of electric field distribution are shown in Figures 4(c) and 4(d). It should be noted that the device only operates effectively when the infinitesimal dipole source is placed right above the geometric center of the device. When the source is moved away from the center, the excited circular wave front is no longer a series of concentric circles centered at the device's center [28]. In this case, the excited circular wave front is unparallel to the ENZ material interface, leading to the spatial phase difference along the arc interface. Thus, the wave fronts of the output wave after propagating through the ENZ region are not plane waves perpendicular to the plane interface, due to the spatial phase difference. Therefore, the source should be placed above the device concentrically to ensure that the input wave front is conformal with the interface and thus the output waves are perpendicular to the output interface.

\section{Conclusion}

In this paper, we theoretically demonstrate that graphene can behave as a novel ENZ material. As one of the applications of ENZ graphene, multiple-beams wave splitter is presented. The beams of the wave splitter are controlled by designing the polygon contours of the ENZ graphene layer. The operation frequency can be designed through changing the gate voltage. Simulation results verify the effectiveness of our design. The ENZ graphene may have important applications in transformational plasmon optics and the proposed wave splitter can be used for generating multiple-beams of SPP waves.

\section{Competing Interests}

The authors declare that they have no competing interests. 


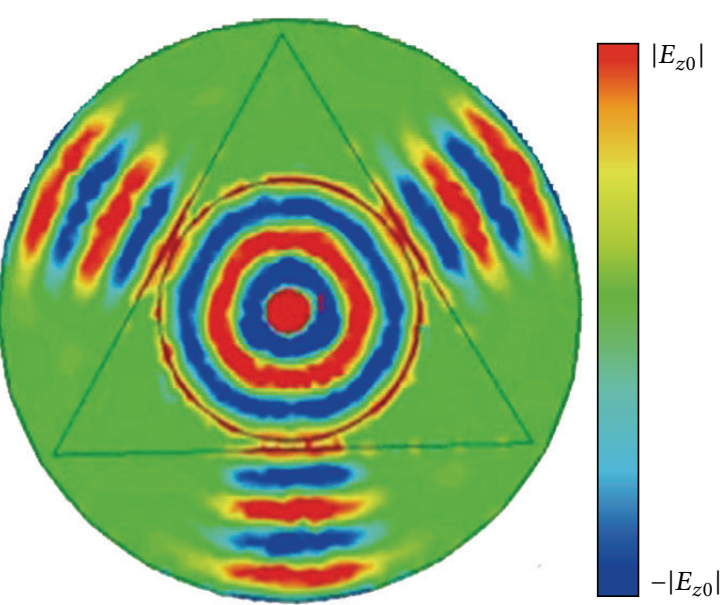

(a)

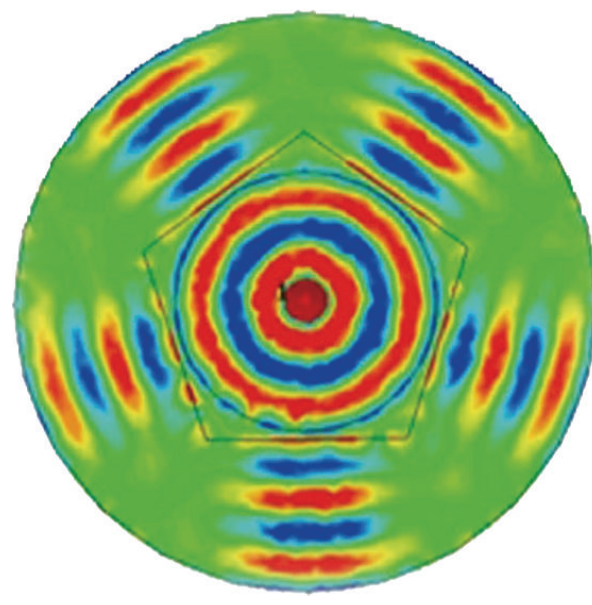

(c)

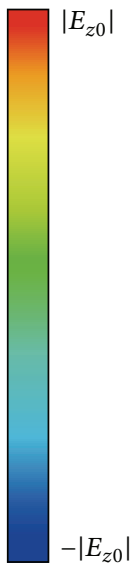

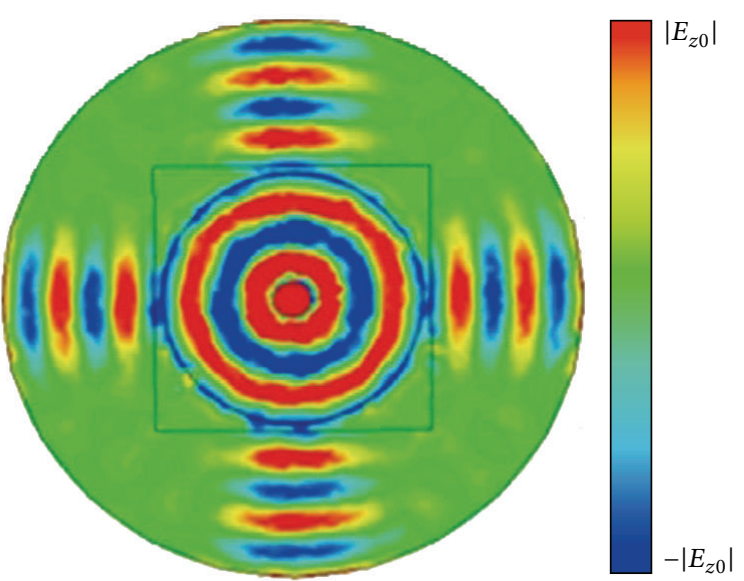

(b)
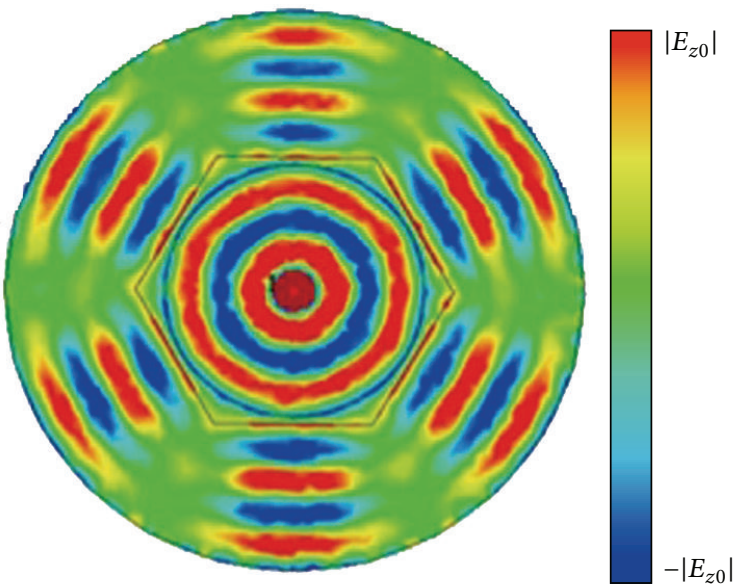

(d)

FIGURE 4: Snapshots of the $z$-component electric field distribution of the TM SPP wave on graphene-based wave splitters with three, four, five, and six beams from (a) to (d), respectively.

\section{Acknowledgments}

This work was partially supported by the National Natural Science Foundation of China [Grant no. 61271057]; the Scientific Research Foundation of Graduate School of Southeast University; the Fundamental Research Funds for the Central Universities; and the Innovation Program for Graduate Education of Jiangsu Province [Grant no. CXLX13_092].

\section{References}

[1] A. Alù, M. G. Silveirinha, A. Salandrino, and N. Engheta, "Epsilon-near-zero metamaterials and electromagnetic sources: tailoring the radiation phase pattern," Physical Review B, vol. 75, no. 15, Article ID 155410, 2007.

[2] R. Liu, Q. Cheng, T. Hand et al., "Experimental demonstration of electromagnetic tunneling through an epsilon-nearzero metamaterial at microwave frequencies," Physical Review Letters, vol. 100, no. 2, Article ID 023903, 2008.
[3] M. Silveirinha and N. Engheta, "Tunneling of electromagnetic energy through subwavelength channels and bends using $\mathcal{E}$ near-zero materials," Physical Review Letters, vol. 97, no. 15, Article ID 157403, 2006.

[4] T. Y. Kim, M. A. Badsha, J. Yoon, S. Y. Lee, Y. C. Jun, and C. K. Hwangbo, "General strategy for broadband coherent perfect absorption and multi-wavelength all-optical switching based on epsilon-near-zero multilayer films," Scientific Reports, vol. 6, Article ID 22941, 2016.

[5] S. Campione, J. R. Wendt, G. A. Keeler, and T. S. Luk, "Near-infrared strong coupling between metamaterials and epsilon-near-zero modes in degenerately doped semiconductor nanolayers," ACS Photonics, vol. 3, no. 2, pp. 293-297, 2016.

[6] S. Lee, T. Q. Tran, M. Kim, H. Heo, J. Heo, and S. Kim, "Angle- and position-insensitive electrically tunable absorption in graphene by epsilon-near-zero effect," Optics Express, vol. 23, no. 26, pp. 33350-33358, 2015.

[7] S. Enoch, G. Tayeb, P. Sabouroux, N. Guérin, and P. Vincent, "A metamaterial for directive emission," Physical Review Letters, vol. 89, no. 21, Article ID 213902, 2002. 
[8] A. Lai, C. Caloz, and T. Itoh, "Composite right/left-handed transmission line metamaterials," IEEE Microwave Magazine, vol. 5, no. 3, pp. 34-50, 2004.

[9] J. G. Rivas, C. Janke, P. H. Bolivar, and H. Kurz, “Transmission of $\mathrm{THz}$ radiation through $\mathrm{InSb}$ gratings of subwavelength apertures," Optics Express, vol. 13, no. 3, pp. 847-859, 2005.

[10] W. G. Spitzer, D. Kleinman, and D. Walsh, "Infrared properties of hexagonal silicon carbide," Physical Review, vol. 113, no. 1, pp. 127-132, 1959.

[11] F. J. G. de Abajo, "Graphene plasmonics: challenges and opportunities," ACS Photonics, vol. 1, no. 3, pp. 135-152, 2014.

[12] Y. Fan, N.-H. Shen, T. Koschny, and C. M. Soukoulis, "Tunable terahertz meta-surface with graphene cut-wires," ACS Photonics, vol. 2, no. 1, pp. 151-156, 2015.

[13] A. K. Geim and K. S. Novoselov, "The rise of graphene," Nature Materials, vol. 6, no. 3, pp. 183-191, 2007.

[14] A. H. Castro Neto, F. Guinea, N. M. R. Peres, K. S. Novoselov, and A. K. Geim, "The electronic properties of graphene," Reviews of Modern Physics, vol. 81, no. 1, pp. 109-162, 2009.

[15] A. K. Geim, “Graphene: status and prospects," Science, vol. 324, no. 5934, pp. 1530-1534, 2009.

[16] A. Vakil and N. Engheta, "Transformation optics using graphene," Science, vol. 332, no. 6035, pp. 1291-1294, 2011.

[17] Y. Fan, Z. Liu, F. Zhang et al., "Tunable mid-infrared coherent perfect absorption in a graphene meta-surface," Scientific Reports, vol. 5, Article ID 13956, 2015.

[18] M. Jablan, H. Buljan, and M. Soljačić, "Plasmonics in graphene at infrared frequencies," Physical Review B, vol. 80, no. 24, Article ID 245435, 7 pages, 2009.

[19] L. A. Falkovsky and A. A. Varlamov, "Space-time dispersion of graphene conductivity," The European Physical Journal B, vol. 56, no. 4, pp. 281-284, 2007.

[20] C. R. Dean, A. F. Young, I. Meric et al., "Boron nitride substrates for high-quality graphene electronics," Nature Nanotechnology, vol. 5, no. 10, pp. 722-726, 2010.

[21] K. S. Novoselov, A. K. Geim, S. V. Morozov et al., "Electric field effect in atomically thin carbon films," Science, vol. 306, no. 5696, pp. 666-669, 2004.

[22] L. A. Falkovsky and S. S. Pershoguba, "Optical far-infrared properties of a graphene monolayer and multilayer," Physical Review B, vol. 76, no. 15, Article ID 153410, 2007.

[23] A. Vakil and N. Engheta, "Fourier optics on graphene," Physical Review B, vol. 85, no. 7, Article ID 075434, 4 pages, 2012.

[24] S. H. Lee, M. Choi, T.-T. Kim et al., "Switching terahertz waves with gate-controlled active graphene metamaterials," Nature Materials, vol. 11, no. 11, pp. 936-941, 2012.

[25] L. Ju, B. Geng, J. Horng et al., "Graphene plasmonics for tunable terahertz metamaterials," Nature Nanotechnology, vol. 6, no. 10, pp. 630-634, 2011.

[26] G. W. Hanson, "Dyadic green's functions for an anisotropic, non-local model of biased graphene," IEEE Transactions on Antennas and Propagation, vol. 56, no. 3, pp. 747-757, 2008.

[27] CST Microwave Studio, CST Computer Simulation Technology, http://www.cst.com/.

[28] Q. Zhao, Z. Xiao, F. Zhang et al., "Tailorable zero-phase delay of subwavelength particles toward miniaturized wave manipulation devices," Advanced Materials, vol. 27, no. 40, pp. 6187-6194, 2015. 

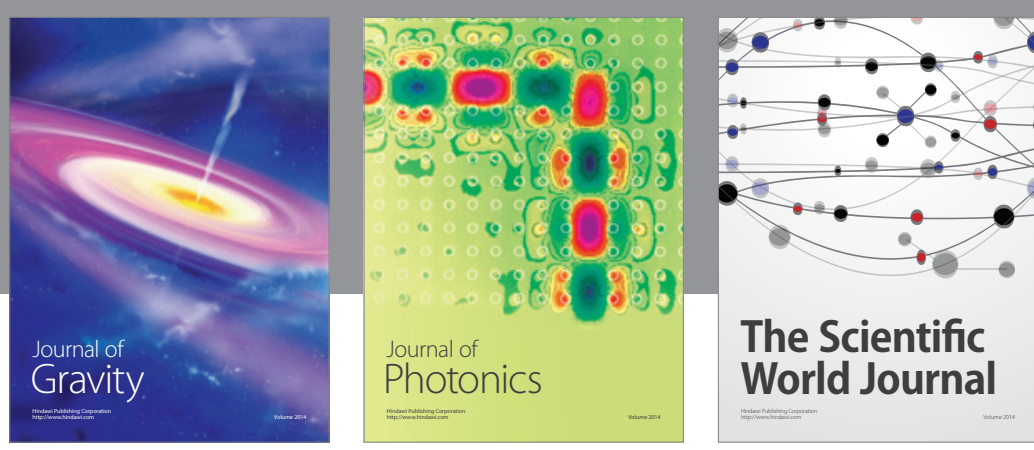

The Scientific World Journal
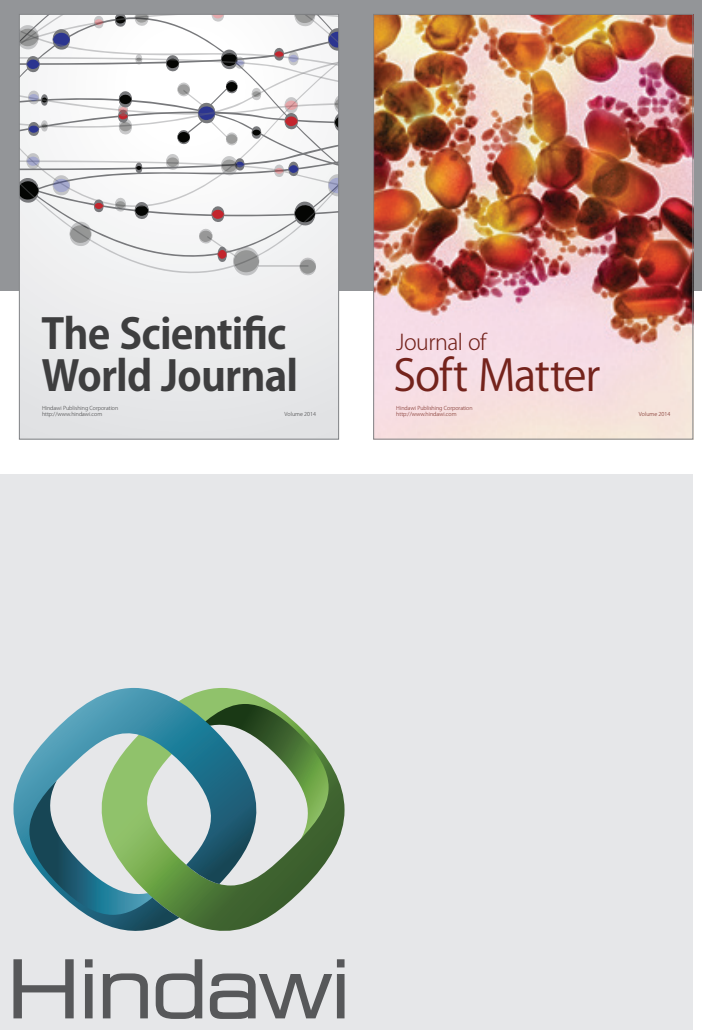

Submit your manuscripts at

http://www.hindawi.com

nternational Journal of

Statistical Mechanics
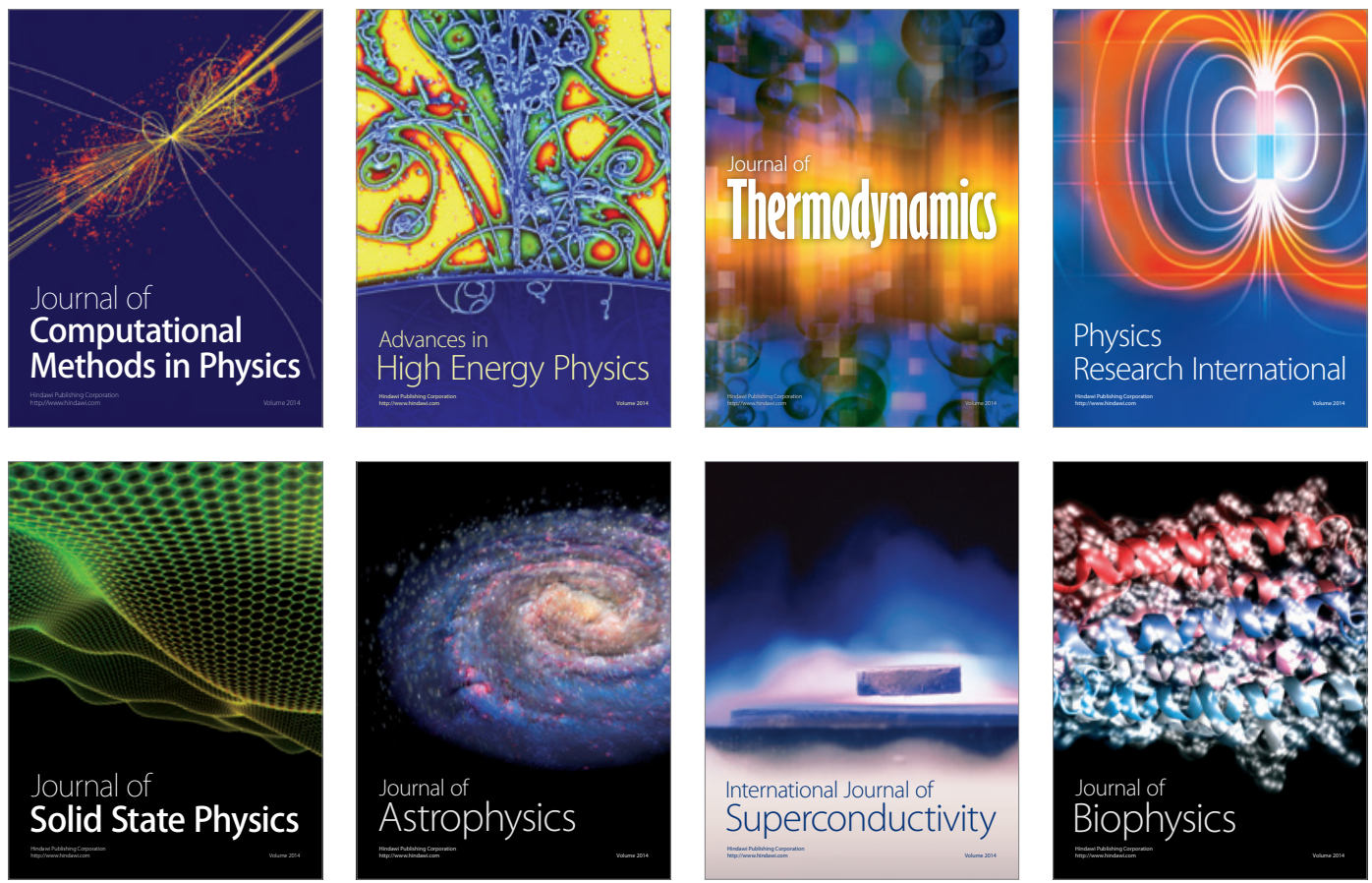
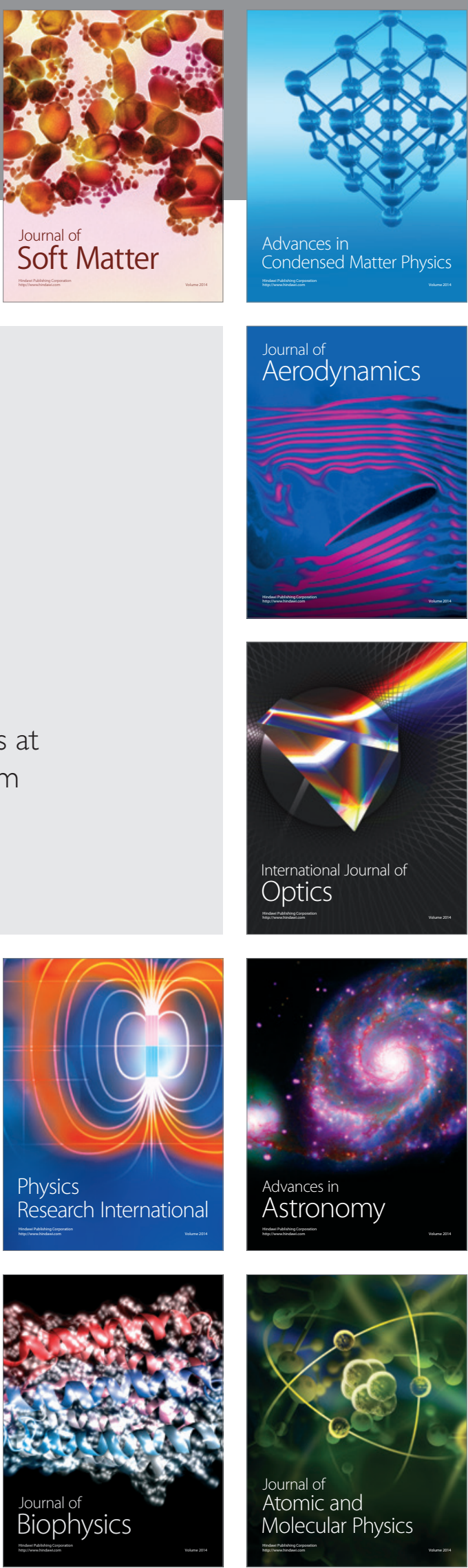